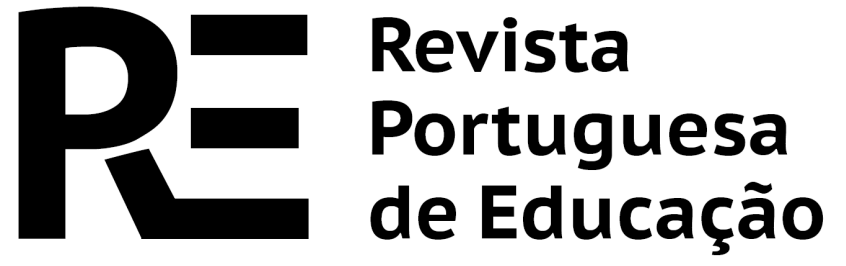

\section{A escolha de uma carreira no ensino em Portugal: Motivos e autoeficácia de estudantes de mestrado}

\section{RESUMO}

Neste estudo analisaram-se os motivos de estudantes para escolherem a carreira docente e respetivas associações com variáveis sociodemográficas, com características do curso de formação de professores e com crenças de autoeficácia. Participaram no estudo 183 estudantes (86\% do género feminino) de cursos de mestrado em ensino de instituições portuguesas, que preencheram um questionário on-line sobre características sociodemográficas, motivos da sua decisão de se tornarem professores e crenças de autoeficácia. Através da análise fatorial, foram derivados quatro fatores subjacentes aos motivos para ser professor: (a) Benefícios da profissão, (b) Influências sociais, (c) Interesse no curso ou nos conteúdos académicos, e (d) Promoção do desenvolvimento das crianças/adolescentes. Foram encontrados valores médios mais elevados nos motivos relacionados com a Promoção do desenvolvimento das crianças/adolescentes e com o Interesse no curso/nos conteúdos académicos. Foram encontradas diferenças estatisticamente significativas entre os motivos de estudantes que optaram por um curso que profissionaliza para a educação pré-escolar e/ou primeiro ciclo e os que optaram por um curso que profissionaliza para o ensino de disciplinas específicas, assim como em função da idade, do género e da autoeficácia. As implicações para a formação de professores são discutidas.

Palavras-chave: Ensino; Motivos intrínsecos; Motivos extrínsecos; Carreira docente

\section{INTRODUÇÃO}

Dados recentes divulgados no relatório Education at a Glance 2018 (Organização para a Cooperação Económica e Desenvolvimento [OCDE], 2018) indicam que, em Portugal, a média de idades da classe docente aumentou significativamente ao longo da última década, sendo uma das mais envelhecidas no conjunto dos países membros da OCDE, nomeadamente em consequência da diminuição de contratações de docentes. Em 2016, apenas
Sílvia Barrosi

Instituto Politécnico do Porto, Portugal

Carla Peixoto ii

Instituto Universitário da Maia, Portugal

Manuela Pessanhaiii

Instituto Politécnico do Porto, Portugal

Tiago Ferreiraiv

Instituto Politécnico do Porto, Portugal

Ana Barrosv

Instituto Politécnico do Porto, Portugal

Ewald Kielvi

Ludwig-Maxmilians-

University of Munich,

Alemanha

Sabine Weißvii

Ludwig-Maxmilians University of Munich, Alemanha

Manuela Keller-

Schneiderviii

Pädagogische Hochschule Zürich, Suiça 
1\% dos professores a lecionar nos ensinos básico ou secundário tinham menos de 30 anos (média OCDE: 11\%) e 38\% tinham 50 ou mais anos (média OCDE: 35\%). Além disso, ao contrário do que acontece em outros países, o ensino não tem sido uma das profissões mais valorizadas socialmente, como é frequentemente transmitido pelos meios de comunicação social ou pelos discursos políticos (considerados, por vezes ambíguos, como referido por Nóvoa em 1999), tendendo os professores portugueses a revelarem baixa satisfação profissional (e.g., Pedro \& Peixoto, 2006). Neste contexto, a procura de cursos de Ensino Superior na área da formação de professores tem sofrido um decréscimo acentuado. A título de exemplo, refira-se que na primeira fase do concurso nacional de acesso ao Ensino Superior, no ano letivo de 2018/2019, apenas foram preenchidas 693 das 1204 vagas disponíveis para a Licenciatura em Educação Básica (Direção-Geral do Ensino Superior, 2018).

Considerando que, em Portugal, vários fatores económicos e sociais podem atualmente desencorajar os estudantes de optarem por cursos de formação de professores, é relevante explorar os seus motivos para desejarem uma carreira na área do ensino. Esta análise é importante para se preparar melhor a sua formação, assim como para se ajustarem as políticas educativas relacionadas com a formação de professores e se melhorar a qualidade do ensino (Heinz, 2015; Lin et al., 2012). Tem sido também realçada a importância dos motivos subjacentes à opção por uma carreira no ensino para se compreender o percurso académico dos estudantes, nomeadamente o seu grau de envolvimento e compromisso no curso e, futuramente, no desempenho profissional (e.g., Fokkens-Bruinsma \& Canrinus, 2012, 2014; Jungert et al., 2014; Sinclair, 2008).

Apesar de se registar, a nível internacional, interesse dos investigadores por esta área (e.g., Fokkens-Bruinsma \& Canrinus, 2014; Heinz, 2015), a investigação realizada em Portugal (e.g., Flores \& Niklasson, 2014) é ainda escassa, sendo necessário realizar mais estudos cuja amostra não se limite a um único contexto institucional, como refere Heinz (2015). Neste sentido, no presente estudo analisaram-se os motivos de estudantes para escolherem mestrados que profissionalizam para o ensino, assim como a sua associação com variáveis sociodemográficas, características do curso de formação de professores (Generalista ou amplo vs. organizado em torno de disciplinas específicas, i.e., de educação pré-escolar e/ou ensino do 1 1. $^{\mathrm{o}}$ ciclo vs. ensino do 2.ำ ciclo ao secundário) e crenças de autoeficácia.

\subsection{MOTIVOS PARA A ESCOLHA DE UMA CARREIRA NO ENSINO}

Em vários domínios da nossa vida, a motivação é definida como o que nos move a fazer algo, o que inclui o início de uma carreira ou o ingresso num curso de formação de professores. Neste âmbito, as motivações podem determinar o que atrai os indivíduos para a área do ensino, por quanto tempo permanecem nessa área ou até que ponto se envolvem ou concentram no curso e na profissão (Sinclair, 2008). Efetivamente, a escolha de uma carreira profissional, incluindo a de docência, implica um processo de tomada de decisão complexo (Bilim, 2014; Weiss \& Kiel, 2013), normalmente sustentada por um conjunto variado de motivos, explorados nas últimas décadas por investigadores em vários países (Azman, 2013). Numa síntese e análise de 41 estudos desenvolvidos em 23 países, Heinz (2015) destacou que os investigadores têm agrupado os motivos para a escolha de uma carreira no ensino essencialmente em três categorias: intrínsecos, extrínsecos e 
altruístas. Os motivos intrínsecos relacionam-se com o gosto pessoal pelo ensino, o interesse específico em ensinar determinado conteúdo e o sentido de vocação. Os motivos altruístas relacionam-se com a possibilidade de contribuir para a sociedade e o sentido de responsabilidade relativamente à educação de crianças/jovens. Os motivos extrínsecos envolvem aspetos do exercício da profissão não diretamente inerentes à docência, como o salário, a estabilidade profissional e o prestígio. Estas definições podem encontrar-se em estudos como os de Azman (2013), Fokkens-Bruinsma e Canrinus (2012), Jungert et al. (2014), Watt e Richardson (2008).

É possível encontrar na literatura outras categorizações, embora bastante menos comuns, como a que faz a distinção entre motivos adaptativos e mal-adaptativos, ou seja, motivos relacionados positiva ou negativamente com o esforço, o envolvimento e o compromisso, respetivamente (Bruinsma \& Jansen, 2010; Fokkens-Bruinsma \& Canrinus, 2012). Além da disparidade da definição das categorias, é necessário ter também em consideração que, em alguns estudos, (a) se verifica uma fragilidade na definição conceptual das diferentes categorias de motivos, surgindo sobreposições maioritariamente entre os motivos intrínsecos e altruístas, muitas vezes misturados ou colapsados, e (b) é incluída informação limitada quanto à validade e fidedignidade dos instrumentos utilizados (Heinz, 2015). Assim, novos estudos devem ter estes aspetos em consideração. Adicionalmente, há a necessidade de incluir também outros motivos que parecem não ter sido considerados na investigação prévia que apresentava aquelas três categorias. Heinz (2015) destaca então que, além dos motivos de natureza intrínseca, altruísta e extrínseca habitualmente considerados, alguns estudos têm vindo a analisar igualmente a influência das crenças relacionadas com a competência para a docência (e.g., Heinz, 2013; Watt \& Richardson, 2008), as experiências prévias de ensino e de aprendizagem (e.g., Heinz, 2013; Watt et al., 2012), a influência de membros da família (e.g., Drudy et al., 2005; Flores \& Niklasson, 2014) e o impacto de fatores socioculturais (e.g., Klassen et al., 2011).

Em geral, a investigação prévia, maioritariamente desenvolvida na Europa, nos EUA e na Austrália, tem mostrado que os estudantes referem várias razões para a sua opção e não um motivo único (Struyven et al., 2013), parecendo os motivos intrínsecos e/ou altruístas ser mais importantes nesse processo de tomada de decisão (e.g., Azman, 2013; Bastick, 2000; FokkensBruinsma \& Canrinus, 2014; Jungert et al., 2014; Moran et al., 2001; Struyven et al., 2013). É importante considerar, porém, que alguns estudos têm indicado variabilidade nos motivos para a escolha da carreira no ensino em função do contexto sociocultural (e.g., Klassen et al., 2011; Kyriacou et al., 1999; Watt \& Richardson, 2012). Ao contrário dos resultados expostos, em países como a Malásia, o Zimbabwe e o Malawi alguns estudos mostraram maior influência de motivos extrínsecos (cf. Heinz, 2015).

Em Portugal, a investigação desenvolvida nesta área não é muito abundante, sendo de destacar o estudo comparativo entre Portugal e a Suécia, desenvolvido por Flores e Niklasson (2014). Ainda que os dados a nível nacional tenham sido recolhidos exclusivamente numa instituição de Ensino Superior portuguesa, os resultados indicaram que as principais influências subjacentes ao interesse no ensino eram o desejo de trabalhar com crianças, a aquisição de conhecimento aprofundado num determinado domínio, o desenvolvimento de competências sociais e o contributo para a sociedade. Parecem, assim, destacar-se os motivos altruístas e intrínsecos na 
escolha feita por estes estudantes portugueses. No entanto, como o estudo foi realizado numa única instituição, surge a necessidade de analisar estas questões com uma amostra mais variada.

\subsection{RELAÇÃO ENTRE OS MOTIVOS, VARIÁVEIS PESSOAIS E CARACTERÍSTICAS DO CURSO}

Além da influência do contexto sociocultural, já referida anteriormente, outros fatores pessoais e os relacionados com o curso podem contribuir para a compreensão da variabilidade existente entre os estudantes no que concerne aos motivos da escolha da carreira no ensino. Alguns estudos apontam para a existência de diferenças em função do género, sendo que as mulheres parecem atribuir maior importância a motivos intrínsecos do que os homens, tendendo estes a privilegiar motivos extrínsecos (e.g., Johnston et al., 1999; Moran et al., 2001; Struyven et al., 2013). Em estudos que analisaram os motivos altruístas, foi encontrada uma tendência para estes serem mais valorizados por mulheres (Jungert et al., 2014). Porém, é de salientar que nem em todos os estudos se encontram diferenças estatisticamente significativas entre homens e mulheres (e.g., Azman, 2013).

A investigação desenvolvida a nível da relação entre a idade e os motivos subjacentes à escolha de uma carreira no ensino, além de escassa, tem produzido resultados inconsistentes, pelo que, com o presente estudo, se pretendia contribuir para clarificar algumas destas associações. Pode ser destacado, por exemplo, o estudo de Moran e colaboradores (2001), no qual se verificou que os estudantes com menos de 25 anos eram mais propensos a interessar-se pelo ensino por gostarem de crianças, enquanto os mais velhos davam maior importância às condições de emprego. Por sua vez, Struyven e colaboradores (2013) não encontraram diferenças significativas em função da idade quanto ao motivo relacionado com a vontade de ajudar as crianças, mas ter boas perspetivas ao nível do emprego foram mais valorizadas por estudantes mais novos ( $\leq 23$ anos).

Alguns estudos têm documentado que as características do curso de formação de professores (e.g., o nível de ensino) são um fator que deve ser estudado. Weiss e Kiel (2013), na Alemanha, compararam os motivos que orientam a escolha da profissão por estudantes em cursos de formação de professores que qualificam para níveis de ensino que em Portugal correspondem ao 1. o e 2. ${ }^{\circ}$ ciclos (até 10-12 anos de idade, dependendo da região) e por estudantes dos cursos que qualificam para o ensino nos níveis seguintes, isto é, no ensino geral, no ensino técnico ou para o prosseguimento de estudos (i.e., "secondary modern", "secondary technical" and "grammar schools"). Foram encontradas diferenças significativas principalmente entre o primeiro grupo e os restantes, sendo os futuros professores do equivalente ao 1..$^{\mathrm{o}}$ e 2. ${ }^{\mathrm{o}}$ ciclos (a) mais orientados por motivos pedagógicos (e.g., gosto de estar com crianças/jovens), idealistas (e.g., para formar/educar pessoas), de promoção/proteção dos alunos em situações especiais (e.g., para apoiar crianças/jovens com situações desfavoráveis), pelas múltiplas possibilidades de planificação, e (b) menos orientados pelo interesse na disciplina/matéria, pela segurança financeira e profissional e por verem o ensino como uma solução de emergência (e.g., porque eu não tinha possibilidade de cumprir a minha primeira escolha profissional). $\mathrm{Na}$ Suécia, Jungert et al. (2014), verificaram que estudantes a frequentar cursos para a docência no nível secundário atribuíram significativamente mais importância a motivos de natureza intrínseca (e.g., Estou muito interessado 
na área em que me vou tornar professor), enquanto estudantes a frequentar cursos destinados ao ensino no nível primário a motivos altruístas (e.g., Como professora, posso dar uma contribuição valiosa para a sociedade). Por sua vez, na Holanda, Fokkens-Bruinsma e Canrinus (2014) verificaram que trabalhar com crianças e adolescentes foi identificado como um dos motivos mais relevantes para a tomada de decisão por estudantes que frequentavam um curso para lecionar no nível de ensino primário (destinado a alunos dos 4 aos 12 anos de idade) e por estudantes que frequentavam um curso para lecionar no nível de ensino secundário (destinado a alunos a partir dos 12 anos), ainda que este motivo se tenha revelado como significativamente mais relevante para o primeiro grupo de estudantes. A influência social surgiu como um dos motivos menos importantes para ambos os grupos. Os autores verificaram que, comparativamente aos estudantes que frequentavam um curso para lecionar no ensino secundário, estudantes que frequentavam um curso para lecionar no ensino "primário" atribuíram significativamente mais importância a aspetos como o estatuto social, o salário e a sua perceção de impacto no futuro das crianças e dos adolescentes.

A crença de autoeficácia parece desempenhar um papel fundamental nos processos motivacionais (e.g., Bandura, 1993), não apenas ao longo do desempenho profissional dos professores (cf., Weiss \& Keil, 2013), mas também na escolha da carreira docente (e.g., Betz \& Hackett, 1981). Contudo, os estudos que exploram a associação entre autoeficácia e motivos para a escolha do curso de profissionalização de professores são pouco abundantes. Pode ser destacado o estudo de Bilim (2014) que encontrou uma associação positiva moderada entre a autoeficácia e os motivos intrínsecos e altruístas, se bem que com estes últimos se trate de uma associação de menor magnitude. Nesse estudo não se encontraram associações relevantes com os motivos extrínsecos, embora outros autores tenham encontrado uma associação negativa, ou seja, estudantes com autoeficácia mais baixa tendem a valorizar mais os motivos extrínsecos na sua opção pela carreira docente (Bruinsma \& Jansen, 2010).

\subsection{O PRESENTE ESTUDO}

Com o presente estudo pretende-se compreender o que motiva os estudantes a optarem por um curso de ensino, num período em que há pouca possibilidade de os recém-formados terem colocação numa escola, contrariamente ao que se verifica em outras realidades (por exemplo, EUA, Inglaterra e Holanda) que experimentam dificuldades em recrutar docentes (e.g., Flores, 2012). Assim, analisaram-se os motivos de estudantes do Ensino Superior Português para escolherem a carreira de professor/educador e verificaram-se as associações com variáveis sociodemográficas (idade, género), com características do curso de formação de professores (generalista/amplo vs. organizado em torno de disciplinas específicas, i.e., de educação pré-escolar e/ou ensino do $1^{\mathrm{o}}$ ciclo vs. ensino do $2^{\mathrm{o}}$ ciclo ao secundário) e com crenças de autoeficácia. 


\section{MÉTODO}

\subsection{PARTICIPANTES}

Os dados utilizados neste estudo fazem parte de um projeto designado Student Teachers' Motives (STeaM). Self-concept and Career Choice Motives of Teacher Students, que foi desenvolvido no âmbito de uma colaboração entre investigadores do Centro de Investigação e Inovação em Educação (Escola Superior de Educação do Politécnico do Porto), da LudwigMaxmilians-Universität München (Alemanha) e da Pädagogische Hochschule Zürich (Suíça).

No presente estudo, participaram 183 estudantes (86\% do sexo feminino) inscritos em diversos cursos de mestrado profissionalizantes na área do ensino, de instituições de ensino superior portuguesas. Para se obter esta amostra, contactaram-se os coordenadores dos cursos de mestrado profissionalizantes em ensino de todo o país, tendo-lhes sido solicitado que divulgassem o projeto junto dos seus mestrandos e os convidassem a preencher o questionário. Foi-lhes enviado o endereço para acederem ao questionário, que estaria disponível durante o primeiro semestre, devido a exigências do projeto em que se insere. No Quadro 1 é apresentada informação sociodemográfica acerca dos estudantes que preencheram o questionário. A maioria dos participantes era do Norte (36\%) ou do centro de Portugal (38\%), e as suas idades variavam entre 20 e 55 anos $(M=26.73, D P$ $=7.49$ ).

Quadro 1

Dados sociodemográficos dos participantes

\begin{tabular}{llcc}
\hline & & $M(D P)$ & $n(\%)$ \\
\hline Idade & & $26.73(7.49)$ & - \\
Género & Masculino & - & $158(86.3)$ \\
Área geográfica & Norte & - & $25(13.7)$ \\
& Centro & - & $66(36.1)$ \\
& Outra & - & $69(37.7)$ \\
Tipo de curso de & Programa generalista (Pré-escolar & - & $47(25.7)$ \\
mestrado & e/ou 1. ${ }^{\circ}$ ciclo do EB) & - & $90(49.2)$ \\
& Programa associado a disciplina(s) & & \\
& (2. ${ }^{\circ}$ ciclo, 3..$^{\circ}$ ciclo, secundário, & - & \\
& ensino de música/artes) & & \\
\hline
\end{tabular}

\subsection{INSTRUMENTOS}

Os dados foram recolhidos através do preenchimento de um conjunto de questionários, que incluía, entre outras informações, dados sociodemográficos (e.g., idade, tipo de curso, região do país onde se situava a instituição de Ensino Superior), motivos da opção pela carreira na educação/no ensino e crenças de autoeficácia.

QUESTIONÁRIO SOBRE OS MOTIVOS DA DECISÃO

DE SE TORNAR PROFESSOR/A OU EDUCADOR/A

Os motivos para a carreira docente foram recolhidos através do Questionário sobre os Motivos da Decisão de se Tornar Professor (QMDTP), desenvolvido por Kiel et al. (2004). Este questionário foi traduzido no âmbito do presente estudo através de um processo com as seguintes fases: (a) tradução inicial, 
(b) revisão da tradução, e (c) breve estudo piloto. 0 estudo piloto foi realizado com estudantes de mestrado que não poderiam participar no estudo, por, entretanto, concluírem os seus cursos. Este processo permitiu verificar se os itens se encontravam redigidos de forma adequada e correspondiam claramente à situação portuguesa. Posteriormente, foram efetuadas algumas alterações na formulação dos itens, para haver um melhor ajuste dos mesmos aos eventuais participantes e seus cursos.

O QMDTP é constituído por 73 itens que avaliam os motivos para a opção por um curso de formação de professores/educadores. Os participantes são convidados a responder, numa escala de 4 pontos $(1=$ discordo totalmente, 2 = discordo ligeiramente, $3=$ concordo ligeiramente, $4=$ concordo totalmente), em que medida ingressaram no curso de formação de professores por cada um dos motivos enunciados (i.e., "Decidi ingressar na formação de professores porque/para..."). A estrutura fatorial originalmente proposta por Keller-Schneider et al. (2018), para os 73 itens do QMDTP, incluía 11 dimensões que avaliam motivos intrínsecos no sentido restrito (quatro dimensões relacionadas com aspetos como a satisfação com o trabalho com crianças/jovens, a preocupação com a promoção do seu desenvolvimento, o interesse no curso e nos conteúdos), motivos intrínsecos no sentido lato (duas dimensões com itens relacionados com a utilidade social), motivos extrínsecos (duas dimensões com aspetos relacionados com segurança profissional e autonomia) e motivos não relacionados com a profissão (três dimensões com itens relacionados com a recomendação efetuada por outras pessoas ou a falta de alternativa). A validade desta estrutura fatorial para a amostra do presente estudo foi inspecionada através da análise fatorial confirmatória (AFC). Este modelo não convergiu, possivelmente devido à complexidade do modelo fatorial e por limitações relacionadas com o tamanho da amostra. Assim, foi desenvolvida uma estrutura fatorial alternativa através de procedimentos de análise fatorial exploratória (AFE) com rotação oblíqua (i.e., promax). Foram derivados 4 fatores, explicando 38\% da variância de 28 itens considerados: (a) Benefícios da profissão, (b) Influências sociais, (c) Interesse no curso ou nos conteúdos académicos, e (d) Promoção do desenvolvimento das crianças / adolescentes. 0 fator "Benefícios da profissão" (8 itens; $\alpha=.80$ ) inclui motivos extrínsecos, predominantemente relacionados com a segurança profissional e o prestígio, assim como com a autonomia na gestão do tempo (e.g., porque os professores/educadores são bem pagos; para, no futuro, ser capaz de conciliar a família e o emprego; porque a profissão tem elevado prestígio social; para ter oportunidade de definir o meu horário de trabalho fora da sala/escola). 0 fator "Influências sociais" (5 itens; $\alpha=.69$ ) inclui motivos extrínsecos relacionados com a recomendação de pessoas significativas, mas também razões relacionadas com a ausência de comprometimento (e.g., por recomendação da minha mãe; porque tenho pouco conhecimento acerca de outros cursos). 0 fator "Interesse no curso/nos conteúdos académicos" (8 itens; $\alpha=.79$ ) inclui motivos intrínsecos, relacionados com os conteúdos específicos do curso e com a área de especialização e/ou recrutamento (i.e., porque estou interessado/a numa ou em várias disciplinas do curso; porque, mesmo que não venha a trabalhar como professor/a, os conteúdos são úteis; porque tenho especial interesse em teorias pedagógicas e psicológicas). Finalmente, o fator "Promoção do desenvolvimento das crianças/adolescentes" ( 6 itens; $\alpha=.75$ ) inclui motivos altruístas, que dizem respeito à preocupação com o desenvolvimento e a aprendizagem das 
crianças/alunos, particularizando também as crianças com dificuldades específicas (i.e., para cuidar de crianças/adolescentes que tenham tido experiências educativas desadequadas; para proteger as crianças/adolescentes de dificuldades no seu desenvolvimento; porque estou muito interessado/a em ensinar/educar). Os restantes itens da escala original foram desconsiderados, uma vez que apresentavam baixa variância ou variância partilhada entre diferentes fatores.

ESCALA DE AUTOEFICÁCIA GERAL

As crenças de autoeficácia foram avaliadas através da versão portuguesa do questionário General Self-Efficacy Scale (GSES; Schwarzer \& Jerusalem, 1995), utilizado anteriormente em Portugal por Araújo e Moura (2011) com boas propriedades psicométricas. Este questionário é constituído por 10 itens (e.g., consigo resolver a maior parte dos problemas, se me esforçar; costumo saber lidar com situações novas), que se organizam numa só dimensão e avaliam o sentimento de competência da pessoa para lidar com situações variadas. Cada item apresentava 4 possibilidades de resposta, de 1 (nada verdadeiro) a 4 (completamente verdadeiro), obtendo-se uma pontuação média calculada com base nas respostas dadas aos 10 itens $(\alpha=$ .88).

\subsection{PROCEDIMENTOS}

RECOLHA DE DADOS

Os dados foram recolhidos durante o primeiro semestre do ano letivo 20152016. 0 questionário foi preenchido on-line, numa plataforma criada para o efeito e que apresentava informação pormenorizada sobre o projeto e sobre a equipa de investigação. 0 consentimento livre e informado foi recolhido online: após a leitura da informação sobre o projeto, os participantes poderiam decidir preencher ou não o questionário, selecionando a respetiva opção, e podiam interromper o seu preenchimento a qualquer momento. Não foram recolhidos dados que permitissem identificar os estudantes nem a instituição que frequentavam. Todos os dados foram recolhidos e arquivados de forma anónima.

\section{ANÁLISES}

Após a análise de indicadores estatísticos descritivos para as variáveis observadas, recorreu-se a modelação de equações estruturais multigrupo para abordar as principais questões de investigação. Utilizou-se uma versão modificada de estimação pelo método de máxima verosimilhança para acomodar desvios à normalidade dos dados, considerando toda a informação disponível (Arbuckle, 1996; Huber, 1967). Foram utilizadas parcelas para definir as variáveis latentes consideradas no modelo: (a) Motivos associados a benefícios da profissão; (b) Influências sociais; (c) Interesse no curso/nos conteúdos académicos; (d) Interesse na promoção do desenvolvimento das crianças/adolescentes; e (e) Autoeficácia.

Numa primeira fase da análise de dados, foi avaliada a adequação do modelo de medida, bem como a sua invariância entre estudantes de cursos destinados à educação de infância e docência do 1.ํ Ciclo (i.e., monodocência) e estudantes de cursos que habilitam para a docência de disciplinas académicas específicas (e.g., Português, Matemática, História, etc.). Para o efeito, foi conduzida uma análise fatorial confirmatória (AFC) e procedimentos para testar a invariância de medida (IM). A AFC é uma técnica 
estatística teoricamente orientada que permite examinar em que medida um hipotético modelo de medida se ajusta aos dados observados. Em síntese, a AFC examina o grau de concordância entre uma matriz de covariações observadas e uma matriz de covariações da população, estimada com base num modelo de medida teórico. Este procedimento pode ser enquadrado numa abordagem de análise multigrupo para determinar se diferentes componentes do modelo de medida, nomeadamente pesos fatoriais (invariância fraca) e interceptos dos itens (invariância forte), são equivalentes entre diferentes grupos de sujeitos (cf. Brown, 2006).

Numa segunda fase das análises estimou-se um modelo de hipóteses, incluindo os tipos de motivos para a carreira docente, como variáveis dependentes, e autoeficácia, género ( 0 = masculino; $1=$ feminino $)$ e idade, como variáveis independentes. Os modelos foram identificados, utilizando o método de codificação dos efeitos, proposto por Little et al. (2006), que permite definir a variância e média dos fatores latentes, respetivamente em função dos pesos fatoriais e interceptos dos itens. Este método permite analisar os parâmetros latentes, utilizando uma métrica comparável entre grupos. Para avaliar o ajustamento do modelo proposto, foi especificado um modelo nulo, no qual, para além dos indicadores ortogonais, eram impostas iguais variâncias e médias entre grupos (Little et al., 2007). Para além do teste de chi-quadrado, o ajustamento do modelo foi avaliado tendo em conta os indicadores: (a) "Root Mean Square Error of Approximation" (RMSEA); (b) "Comparative Fix Index" (CFI); e (c) "Standardized Root Mean Square Residual" (SRMR). Valores de RMSEA inferiores a 0.05 , de CFI superiores a 0.95 e de SRMR inferiores a 0.10 consideraram-se indicativos de bom ajustamento do modelo (Schweizer, 2010). As análises foram conduzidas no R (R Core Team, 2013), usando o pacote "lavaan” (Rosseel, 2012).

\section{RESULTADOS}

Examinaram-se as estatísticas descritivas para as variáveis em estudo. As médias, os desvios-padrão e as correlações são apresentadas nos Apêndices A (estatística descritiva para a amostra total) e B (estatística descritiva por grupos de estudantes). Como se pode verificar, os motivos que colheram maior concordância, com médias de 3.16 e 2.83, respetivamente, foram os que se relacionam com a preocupação com o desenvolvimento de crianças e adolescentes e com o interesse nos conteúdos de lecionação. As características relacionadas com a profissão tiveram um valor médio que equivale a discordo ligeiramente e as influências de outras pessoas tiveram a média mais baixa, próxima do valor 1 (= discordo totalmente).

0 modelo de medida subjacente ao presente estudo incluía variáveis latentes, analisando motivos dos estudantes para a carreira docente (i.e., motivos associados aos benefícios da profissão, influências sociais, interesse no curso/nos conteúdos académicos e interesse na promoção do desenvolvimento das crianças/adolescentes), bem como as suas crenças de autoeficácia. Na AFC, estas cinco variáveis estavam correlacionadas livremente. Este modelo proporcionou um bom ajustamento aos dados, $\chi^{2}(80)=110.449, p=0.014, R M S E A=0.046, C F I=0.969, S R M R=0.053$. Após a análise do ajustamento, foi conduzido um conjunto de procedimentos analíticos sequenciais para verificação da invariância do modelo de medida 
em dois grupos de estudantes: estudantes de cursos destinados à educação de infância e docência do 1. ${ }^{\circ}$ Ciclo e estudantes de cursos que habilitam para o ensino de disciplinas académicas específicas. Estes procedimentos consistem na comparação de modelos aninhados progressivamente mais constritos. Primeiro, a invariância fraca foi testada comparando o modelo livre (Modelo A) com um modelo em que a igualdade dos pesos fatoriais foi imposta entre grupos (Modelo B). Segundo, a invariância forte foi testada comparando o Modelo B com um modelo mais constrito, em que, para além dos pesos fatoriais, também os interceptos dos itens foram igualados entre grupos. Os resultados dos procedimentos de IM, apresentados no Quadro 2, comprovam que o modelo de medida é invariante entre os dois grupos de estudantes, tanto ao nível dos pesos fatoriais, $\Delta \chi^{2}(10)=11.65, p=.309, \Delta C F I$ $=0.000$, como dos interceptos dos itens, $\Delta \chi^{2}(10)=8.71, p=.560, \Delta C F I=-$ 0.002 . Assim, estes resultados sugerem, por um lado, que os diferentes itens da escala apresentam pesos fatoriais semelhantes nos dois grupos de estudantes e, por outro lado, que as médias dos diferentes itens não são enviesadas pela pertença a um dos dois grupos de estudantes considerados.

Quadro 2

Indicadores de ajustamento para modelos de AFC aninhados e teste de IM multigrupo

\begin{tabular}{|c|c|c|c|c|c|c|c|c|}
\hline $\begin{array}{l}\text { Modelo } \\
\text { testado }\end{array}$ & $\chi^{2}(\mathrm{df})$ & RMSEA & CFI & SRMR & $\begin{array}{c}\text { Modelo } \\
\text { comparado }\end{array}$ & $\Delta \chi^{2}(\Delta \mathrm{df})$ & $C F I$ & Invariante? \\
\hline Modelo Nulo & $\begin{array}{c}1332.87 \\
(240)^{* *}\end{array}$ & --- & --- & --- & --- & --- & --- & --- \\
\hline $\begin{array}{l}\text { A Invariância } \\
\text { configuracional }\end{array}$ & $\begin{array}{l}204.89 \\
(160)^{*}\end{array}$ & .055 & .959 & .068 & --- & --- & --- & Sim \\
\hline $\begin{array}{l}\text { B Invariância } \\
\text { fraca }\end{array}$ & $\begin{array}{l}216.54 \\
(170)^{* *}\end{array}$ & .055 & .957 & .078 & A & $\begin{array}{c}11.65 \\
(10)\end{array}$ & 0.002 & Sim \\
\hline $\begin{array}{l}\text { C Invariância } \\
\text { forte }\end{array}$ & $\begin{array}{l}225.25 \\
(190)^{*}\end{array}$ & .052 & .959 & .079 & B & $8.71(10)$ & -0.002 & Sim \\
\hline
\end{tabular}

Nota. ${ }^{*} p<.05,{ }^{* *} p<.01$

No Quadro 3 apresentam-se as médias e os desvios padrão estimados para as cinco variáveis latentes consideradas. Estes parâmetros foram estimados para os dois grupos de estudantes, impondo invariância de medida forte. Estudantes de cursos que preparam para o pré-escolar e $1^{\mathrm{o}}$ ciclo apresentam valores médios de interesse no curso/nos conteúdos académicos inferiores aos de estudantes de cursos centrados em tópicos académicos específicos, $\operatorname{Wald}(1)=8.79, p=0.003, d=0.53$. A média do interesse na promoção do desenvolvimento das crianças/adolescentes é maior no primeiro grupo do que no grupo de estudantes de cursos centrados em disciplinas específicas, Wald $(1)=25.25, p<0.001, d=0.90$. As correlações entre todas as variáveis latentes são também apresentadas no Quadro 3. 
Quadro 3

Médias, desvios padrão e correlações estimadas para as variáveis latentes

\begin{tabular}{|c|c|c|c|c|c|c|c|c|}
\hline & \multicolumn{2}{|c|}{$M(D P)$} & & \multirow[b]{2}{*}{1} & \multirow[b]{2}{*}{2} & \multirow[b]{2}{*}{3} & \multirow[b]{2}{*}{4} & \multirow[b]{2}{*}{5} \\
\hline & $\begin{array}{c}\text { Generalista } \\
\text { (Ji e } 1 .^{\circ} \text { ciclo) }\end{array}$ & Disc. específicas & & & & & & \\
\hline \multicolumn{9}{|l|}{$T 1$} \\
\hline 1. Benefícios prof. & $1.73(0.53)$ & $1.89(0.54)$ & $\operatorname{Wald}(1)=3.42$ & --- & $.73^{* *}$ & $.45^{* *}$ & .17 & $.35^{* *}$ \\
\hline 2. Inf. sociais & $1.36(0.44)$ & $1.34(0.32)$ & $\operatorname{Wald}(1)=0.73$ & $.53^{* *}$ & --- & $.42^{* *}$ & .17 & $.32^{* *}$ \\
\hline 3. Int. conteúdos & $2.69(0.58)$ & $2.96(0.48)$ & $\operatorname{Wald}(1)=8.79^{* *}$ & .25 & .07 & --- & $.72^{* *}$ & $.47^{* *}$ \\
\hline 4. Prom. desenv. & $3.36(0.28)$ & $2.98(0.53)$ & Wald $(1)=25.25^{* *}$ & .05 & -.10 & $.80^{* *}$ & --- & .32 \\
\hline 5. Autoeficácia & $3.00(0.50)$ & $2.98(0.44)$ & $\operatorname{Wald}(1)=0.11$ & .17 & -.22 & $.41^{* *}$ & $.31^{*}$ & --- \\
\hline
\end{tabular}

Nota. Valores de correlações para o grupo $\mathrm{Jl}$ e $1 .^{\circ}$ ciclo são apresentados acima da diagonal; Valores de correlações para o grupo Disc. Específicas são apresentados abaixo da diagonal; * $p<.05,{ }^{* *} p<.01$.

Para examinar os efeitos concorrentes entre idade, género e autoeficácia dos estudantes sobre os diferentes motivos para a docência, foi testado um modelo de equações estruturais incluindo idade, género e autoeficácia como variáveis preditoras e Benefícios da profissão, Influências sociais, Promoção do desenvolvimento das crianças/adolescentes e Interesse no curso/nos conteúdos académicos como variáveis dependentes (Fig. 1). Foi adotada uma abordagem analítica multigrupo para investigar em que medida os diferentes parâmetros estimados no modelo são equivalentes nos dois grupos de estudantes. A análise compreendeu a testagem e comparação dos seguintes modelos aninhados: (a) Modelo com todos os parâmetros igualados entre grupos; (b) Modelo com todos os parâmetros livremente estimados nos dois grupos; e (c) Modelo mais parcimonioso e com melhores indicadores de ajustamento, combinando parâmetros igualados e livremente estimados nos dois grupos. 0 modelo final, com parâmetros não significativos aparados, revelou um muito bom ajustamento aos dados, $\chi^{2}(237)=271.278, p=0.062$, $R M S E A=0.040, C F I=0.973, S R M R=0.083$. Estes indicadores de ajustamento sugerem que o modelo final, que inclui parâmetros estimados livremente com parâmetros igualados entre grupos, representa bem e de forma parcimoniosa os dados observados.

A Figura 1 apresenta os coeficientes estandardizados para este modelo final. Para o grupo de estudantes de cursos destinados à educação pré-escolar e $1^{0}$ ciclo, este modelo explica $14 \%$ da variância dos motivos ligados aos benefícios da profissão, 8\% da variância em influências sociais na escolha da profissão, $11 \%$ da variância do interesse na promoção do desenvolvimento das crianças/adolescentes e 19\% da variância do interesse no curso/nos conteúdos académicos. Para o grupo de estudantes de cursos para a docência de disciplinas académicas específicas, este modelo explica $12 \%$ da variância dos motivos ligados aos benefícios da profissão, 10\% da variância em influências sociais na escolha da profissão, $16 \%$ da variância do interesse na promoção do desenvolvimento das crianças/adolescentes e 19\% da variância do interesse no curso/nos conteúdos académicos. Em ambos os grupos de estudantes, verificou-se um efeito positivo da autoeficácia dos estudantes no seu interesse na promoção do desenvolvimento das crianças/adolescentes ( $b=0.342, p=0.011,95 \% C I$ [0.079; 0.605]) e no curso/nos conteúdos académicos $(b=0.480, p<0.001,95 \%$ CI [0.231; 0.728]). 
No grupo de estudantes de cursos destinados à educação préescolar e docência do 1. o ciclo foi observado um efeito negativo da autoeficácia sobre as influências sociais $(b=-0.285, p=0.040,95 \%$ CI [0.557; -0.013]) na decisão de se tornar professor, bem como um efeito positivo da idade sobre o interesse na promoção do desenvolvimento das crianças/adolescentes ( $b=0.342, p=0.011,95 \%$ CI [0.079; 0.605]). Verificouse ainda, neste grupo, que estudantes do sexo feminino apresentam significativamente menor motivação associada aos benefícios da profissão $(b$ $=-0.405, p=0.008,95 \%$ CI [-0.703; -0.107]).

No grupo de estudantes de cursos para a docência de disciplinas académicas específicas verificou-se um efeito positivo da autoeficácia sobre os motivos ligados aos benefícios da profissão $(b=0.360, p=0.010,95 \% C I$ $[0.085 ; 0.636])$ e influências sociais para a escolha do curso $(b=0.327, p=$ $0.012,95 \%$ CI [0.073; 0.581]). Neste grupo foi ainda observado um efeito negativo da idade sobre o interesse na promoção do desenvolvimento das crianças/adolescentes $(b=-0.269, p=0.030,95 \%$ CI $[-0.511 ;-0.027])$.

Figura 1

Modelo de equações estruturais para examinar os efeitos da autoeficácia, sexo e idade sobre os motivos para a decisão de se tornar professor

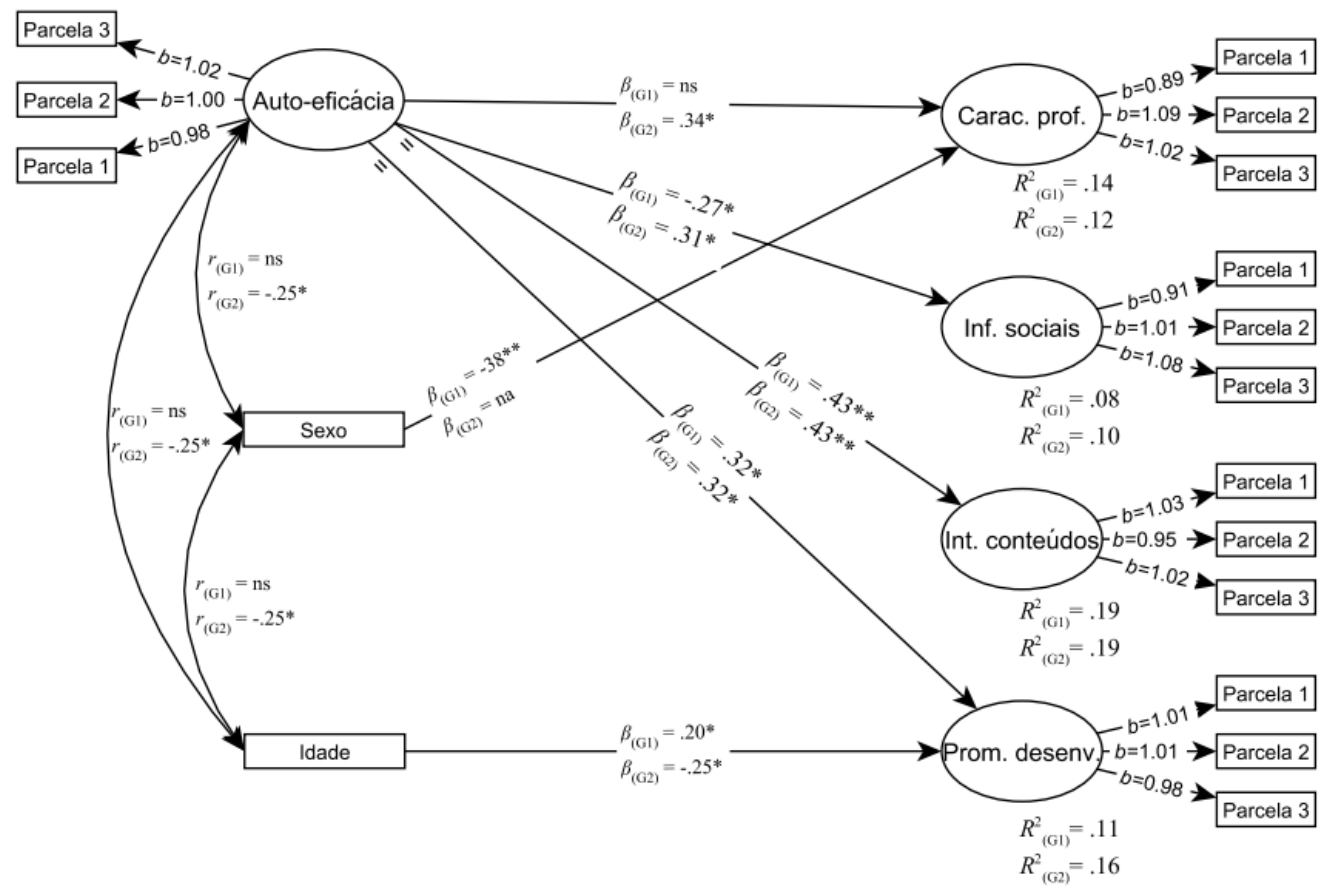

${ }^{*} p<.05 . ; * *<.01$

\section{DISCUSSÃO}

No presente estudo analisaram-se os motivos de estudantes do Ensino Superior para escolherem a carreira de professor/educador de infância em Portugal, num momento que era caracterizado pela baixa empregabilidade nesta área. Como referido previamente, há poucos professores com menos de 30 anos de idade a lecionarem nos diversos níveis de ensino (OCDE, 2018), pelo que nos interessava perceber que razões levam atualmente as pessoas a optarem por estas profissões no nosso país. 
Considerando que a estrutura fatorial original do questionário utilizado não convergiu nas análises efetuadas com a amostra do presente estudo, procedeu-se a uma análise fatorial exploratória que permitiu identificar quatro fatores, que explicavam 38\% da variância: (a) Benefícios da profissão, (b) Influências sociais, (c) Interesse no curso/nos conteúdos académicos, e (d) Promoção do desenvolvimento das crianças/adolescentes. Apesar da variedade de categorizações presente na literatura, esta solução fatorial é coerente com as opções de vários autores (e.g., Azman, 2013; Jungert et al., 2014; Watt \& Richardson, 2008), correspondendo os primeiros dois a motivos tendencialmente extrínsecos, o terceiro a motivos intrínsecos e o quarto a motivos altruístas. Assim, apesar de este estudo ter sido realizado com uma amostra relativamente modesta, estes resultados suportam a opção por utilizar este questionário que poderá ser utilizado com alguma segurança em estudos posteriores. Indicam ainda que, no contexto português, poderá ser utilizada uma versão mais curta do instrumento, com vantagens evidentes no tempo que os/as participantes despendem no preenchimento do questionário, o que pode levar a que haja maior rigor no seu preenchimento e mais participantes a aceitarem responder.

Neste conjunto de estudantes portugueses, foram encontrados valores mais elevados nos motivos que se incluíram nos fatores Promoção do desenvolvimento das crianças/adolescentes e Interesse no curso/nos conteúdos académicos, ou seja, em motivos intrínsecos e altruístas. Como referem Struyven e colaboradores (2013), os estudantes tendem a valorizar vários motivos na escolha do curso de formação de professores, e não apenas um tipo de motivos, o que parece ser o caso também em investigações com estudantes em Portugal. Considerando ainda a globalidade da amostra, as influências sociais e os benefícios da profissão ou da carreira (relacionadas com a segurança profissional e a autonomia no exercício das funções), que são motivos extrínsecos, foram menos indicadas pelos participantes para escolherem a carreira docente. Os estudantes indicaram mesmo uma baixa influência de outras pessoas na sua decisão, com um valor médio muito próximo de 1 (= discordo totalmente), o que é concordante com outros estudos (e.g., Fokkens-Bruinsma \& Canrinus, 2014). Os resultados médios bastante baixos na dimensão dos benefícios da profissão (próximos de $2=$ discordo ligeiramente) parecem-nos coerentes com a atual situação portuguesa, caracterizada por escassas ofertas de emprego na área do ensino e o baixo estatuto profissional desta carreira. Note-se também que em vários estudos tem sido encontrada a mesma tendência, com apenas algumas exceções em países culturalmente bastante diferentes (ver, por exemplo, a síntese da literatura efetuada por Heinz, 2015). Se, por um lado, é interessante que os estudantes estejam motivados para a aprendizagem e que o seu envolvimento possa ser despoletado pelo interesse no curso e pelos efeitos sociais das profissões na área do ensino, por outro lado, estes resultados também poderão indiciar que reconhecem que as condições no mercado atual não são favoráveis ou atrativas. Estas questões poderão ser analisadas com mais detalhe em estudos futuros e devem merecer atenção por parte de decisores políticos e de responsáveis das instituições de ensino, particularmente quando se evidencia que há escassez de candidatos em muitos destes cursos.

Ademais, é de salientar que os motivos intrínsecos são considerados aspetos cruciais da formação dos professores e um importante ponto de análise para compreender melhor questões como compromisso, 
satisfação no trabalho, automotivação e perceções dos estudantes relacionadas com o ensino, a aprendizagem e a formação (Flores, 2012; Fokkens-Bruinsma \& Canrinus, 2012, 2014; Jungert et al., 2014). Deste modo, e apesar de ter vindo a diminuir a procura de cursos que profissionalizam para o ensino, os estudantes que ingressam nestes cursos, a avaliar por esta amostra, indicam que a sua decisão se fundamenta em motivos que têm sido indicados como mais favoráveis à sua formação. Assim, é relevante que os formadores de professores possam mobilizar estrategicamente este interesse, de forma a potenciar o envolvimento e a satisfação dos estudantes ao longo do curso, assim como a construção da sua identidade profissional.

Seguindo os procedimentos de outros estudos recentes (e.g., Fokkens-Bruinsma \& Canrinus, 2014; Jungert et al., 2014; Weiss \& Kiel, 2013), no presente estudo foram comparados os motivos para a escolha da carreira na docência de dois grupos de estudantes: os que optaram por um curso que profissionaliza para a educação pré-escolar e/ou 1. ciclo e os que optaram por um curso que profissionaliza para o ensino de disciplinas específicas, quer seja ao nível do ensino básico (até ao 9.. ano de escolaridade) ou do ensino secundário (10. $\stackrel{\mathrm{o}}{\text { a }} 12 .^{\mathrm{o}}$ ano). Não foram encontradas diferenças no que se refere a motivos considerados extrínsecos. Por outro lado, verificou-se que os estudantes do primeiro tipo de cursos apresentavam médias mais elevadas no que se refere ao interesse na promoção do desenvolvimento das crianças/adolescentes do que o grupo de estudantes de cursos centrados em tópicos académicos específicos. Por outro lado, na educação pré-escolar/1.ํㅜ ciclo foram encontrados valores médios de interesse no curso/nos conteúdos académicos inferiores aos de estudantes de cursos centrados em tópicos académicos específicos. Estas diferenças nas pontuações nos motivos intrínsecos vão no mesmo sentido das que foram encontradas em estudos realizados noutros países (e.g., Fokkens-Bruinsma \& Canrinus, 2014, na Holanda; Weiss \& Kiel, 2013, na Alemanha). Por exemplo, Weiss e Kiel (2013) verificaram que, comparativamente a outros níveis de ensino, futuros professores dos primeiros anos de ensino revelaram-se mais orientados por motivos pedagógicos, idealistas, de promoção/proteção dos alunos em situações especiais, pelas múltiplas possibilidades de planificação, e menos orientados pelo interesse na disciplina/matéria, pela segurança financeira e profissional e por falta de alternativa. Apesar de, nesse estudo, os motivos serem em maior número e mais específicos, os primeiros apresentam semelhança aos motivos intrínsecos e altruístas, e os últimos aos extrínsecos. Note-se que Weiss e Kiel (2013) encontraram também diferenças nos motivos extrínsecos, o que não foi o caso no presente estudo.

As diferenças encontradas em função do curso e, consequentemente, da área de ensino, parecem-nos consistentes com os planos de estudo dos mestrados profissionalizantes e com a própria designação do curso, que já se orienta para uma determinada área específica (e.g., Mestrado em Ensino de Português no 3. Ciclo do Ensino Básico e no Ensino Secundário; Mestrado em Ensino de Biologia e Geologia no 3. Ciclo do Ensino Básico e no Ensino Secundário). Por um lado, parece-nos importante salientar que os motivos relacionados com os conteúdos centrais do plano de estudos são frequentemente considerados aspetos importantes para o desenvolvimento profissional e a motivação global durante o curso e o desempenho da profissão, o que parece estar mais assegurado no contexto dos cursos organizados em torno de uma disciplina específica. Assim, poderá ser importante que os estudantes dos cursos de mestrado em educação pré- 
escolar e ensino no 1.ํ ciclo também desenvolvam uma identidade profissional apoiada no conhecimento científico, no que se refere a conteúdos e metodologias, e não apenas relacionada com uma preocupação genérica com as crianças ou com uma comunicação pragmática do conhecimento (Weiss \& Keil, 2013). Note-se que o valor médio relativo ao interesse pelos conteúdos do curso dos estudantes da educação pré-escolar/1. ciclo foi próximo de 3, o que correspondia a "ligeiramente importante". Assim, é importante que este interesse pelos conteúdos do curso se desenvolva desde o início dos seus estudos, e que não se espere que surja apenas ao longo das suas carreiras. Há estudos em que se verificou que pessoas muito motivadas por razões consideradas mais idealistas, com subvalorização de outros aspetos, tendiam a abandonar mais o ensino e a sentir maior frustração no contacto com a realidade, principalmente quando os contextos não eram tão favoráveis como eles imaginavam (e.g., Schmitz \& Leidl, 1999).

Por outro lado, é esperado que os estudantes com formação organizada em torno de áreas disciplinares específicas, e que na maior parte dos casos correspondem aos grupos de recrutamento nos concursos de professores, adquiram também conhecimentos e competências pedagógicas que lhes permitam desenvolver um ensino de qualidade, relacionados com motivos incluídos em dimensões como a Promoção do desenvolvimento dos alunos. Assim, parece emergir a necessidade de um cuidado especial nestes cursos, pois os estudantes parecem estar mais motivados pela oportunidade de aprofundarem os conhecimentos específicos da sua área disciplinar. Seria positivo que esta responsabilidade não ficasse apenas a cargo de cada docente do Ensino Superior, individualmente, mas que a legislação que enquadra os planos de estudos dos cursos que profissionalizam para o ensino contemplasse também a necessidade de se terem em conta estes aspetos na definição das Unidades Curriculares/disciplinas dos cursos e respetivos conteúdos.

Para cada um dos grupos de estudantes referidos, foram também exploradas associações entre os diversos motivos de escolha da carreira, variáveis sociodemográficas (idade, género) e crenças de autoeficácia. Nos cursos destinados à educação de infância e docência do primeiro ciclo foi observado que estudantes com crenças de autoeficácia mais elevadas tendiam a indicar maior concordância com motivos relacionados com o seu interesse na promoção do desenvolvimento das crianças e o interesse no curso/nos conteúdos académicos. Neste grupo, tendiam a ser os estudantes com crenças de autoeficácia mais baixas a indicar pontuações mais elevadas nas influências sociais na decisão de se tornar professor. Associações positivas entre crenças de autoeficácia e motivos intrínsecos e altruístas também foram encontradas no estudo de Bilim (2014). No presente estudo, estudantes mais velhos tendiam a manifestar que o interesse na promoção do desenvolvimento das crianças/adolescentes tinha tido uma maior relevância na sua decisão. Verificou-se ainda que estudantes do género feminino apresentavam menor motivação associada aos benefícios da profissão, ou seja, revelavam uma menor motivação extrínseca, o que coincide com resultados obtidos anteriormente (e.g., Struyven et al., 2013).

No grupo de estudantes de cursos para a docência de disciplinas académicas específicas verificou-se um efeito positivo da autoeficácia sobre o seu interesse na promoção do desenvolvimento das crianças, o interesse no curso/nos conteúdos académicos, os motivos ligados aos benefícios da profissão e influências sociais para a escolha do curso. Neste grupo foi ainda 
observado que tendiam as ser as pessoas mais novas a indicar uma maior influência do interesse em promover o desenvolvimento das crianças/adolescentes na escolha do curso, o que é concordante com o estudo de Moran e colaboradores (2001) e pode indicar a hipótese de que com o avançar da idade as pessoas ficam ainda mais focadas no tema específico que pretendem ensinar. A influência da idade nas escolhas dos estudantes carece de uma investigação mais aprofundada noutros projetos, como se pode verificar pela existência de resultados discrepantes (cf., Moran et al., 2001; Struyven et al., 2013).

$\mathrm{Na}$ interpretação dos resultados apresentados, devem ser tidas em conta algumas limitações deste estudo. Uma das limitações relaciona-se com o processo de obtenção da amostra. Como foi descrito, o convite para preenchimento dos questionários foi dirigido aos coordenadores de cursos de mestrado que profissionalizam para o ensino e os questionários foram preenchidos por estudantes dos professores que acederam ao nosso convite. 0 facto de o questionário estar disponível durante poucos meses (i.e., primeiro semestre do ano letivo), devido a exigências do projeto internacional em que este estudo se insere, pode ter limitado o número de participantes. De qualquer modo, salientamos que, para a condução deste estudo, foram utilizados procedimentos robustos de análise estatística que, entre outros aspetos, permitem testar diversas hipóteses enquanto se controla o erro associado à medida. Outra limitação refere-se à impossibilidade de controlar variáveis que, no contexto do ensino superior português, poderiam ter relevância, como o nível socioeconómico e/ou tipo de instituição que os estudantes frequentam (i.e., privada vs pública). Estas variáveis não foram consideradas no presente projeto de investigação, pelo que estudos posteriores poderão ter em consideração a necessidade de as controlar de modo a poderem ser exploradas outras associações e, inclusivamente, a poder ser explicada uma percentagem mais elevada de variância dos resultados.

Este estudo foi realizado num momento em que, a nível nacional, persistia a dificuldade em conseguir colocação numa escola pública, especialmente no 1. ciclo do Ensino Básico e na educação pré-escolar. Dado que parece existir uma tendência deste panorama se alterar, começando a ser difícil recrutar professores para algumas zonas do país e em alguns grupos de recrutamento, uma possibilidade interessante para futuros estudos será analisar em que medida se vão modificando as motivações dos estudantes em função dessas alterações no mercado de trabalho.

\section{AGRADECIMENTOS}

O projeto apresentado neste artigo foi desenvolvido no âmbito de uma colaboração entre investigadores da Ludwig-Maxmilians-Universität München (Alemanha), da Pädagogische Hochschule Zürich (Suíça) e do Centro de Investigação e Inovação em Educação (inED), da Escola Superior de Educação do Instituto Politécnico do Porto, que é financiado por fundos nacionais através da FCT - Fundação para a Ciência e a Tecnologia, I.P., no âmbito do projeto UIDB/05198/2020. 


\section{REFERÊNCIAS}

Araújo, M., \& Moura, O. (2011). Estrutura factorial da General Self-Efficacy Scale (Escala de Auto-Eficácia Geral) numa amostra de professores portugueses. Laboratório de Psicologia, 9(1), 95-105.

Arbuckle, J. L. (1996). Full information estimation in the presence of incomplete data. In G. A. Marcoulides \& R. E. Schumacker (Eds.), Advanced structural equation modeling (pp. 243-277). Lawrence Erlbaum Associates.

Azman, N. (2013). Choosing teaching as a career: Perspectives of male and female malaysian student teachers in training. European Journal of Teacher Education, 36(1), 113-130. https://doi.org/10.1080/02619768.2012.678483

Bandura, A. (1993). Perceived self-efficacy in cognitive development and functioning. Educational Psychologist, 28, 117-148.

Bastick, T. (2000). Why teacher trainees choose the teaching profession: Comparing trainees in metropolitan and developing countries. Journal of Personality and Social Psychology, 80(5), 706-722. https://doi.org/10.1023/A:1004090415953

Betz, N. E., \& Hackett, G. (1981). The relationship of career-related selfefficacy expectations to perceived career options in college women and men. Journal of Counseling Psychology, 28(5), 399-410. https://doi.org/10.1037/00220167.28.5.399

Bilim, I. (2014). Pre-service elementary teachers' motivations to become a teacher and its relationship with teaching self-efficacy. Procedia - Social and Behavioral Sciences, 152, 653-661.

Brown, T. A. (2006). Confirmatory factor analysis for applied research. New York, NY, US: The Guilford Press.

Bruinsma, M., \& Jansen, E. P. W. A. (2010). Is the motivation to become a teacher related to pre-service teachers' intentions to remain in the profession?. European Journal of Teacher Education, 33(2), 185-200.

https://doi.org/10.1080/02619760903512927

Direção-Geral do Ensino Superior (2018). Ingresso no ensino superior público, 2018-2019. República Portuguesa, Ciência Tecnologia e Ensino Superior. https://www.dges.gov.pt/coloc/2018/nota_cna18_1f_1.pdf.

Flores, M. A. (2012). A opção por um curso de ensino em tempos desafiadores: Motivações e expectativas de alunos futuros professores. In M. A. Cavalcante, A. F. de Freitas, L. C. V. Pizzi, N. L. F. Fumes, A. Lopes \& M. L. Q. Freitas (Eds.), Formação docente em contextos de mudanças (pp. 23-40). UFAL.

Flores, M. A., \& Niklasson, L. (2014). Why do student teachers enrol for a teaching degree? A study of teacher recruitment in Portugal and Sweden. Journal of Education for Teaching, 40(4), 328-343. https://doi.org/10.1080/02607476.2014.929883

Fokkens-Bruinsma, M., \& Canrinus, T. E. (2012). Adaptive and maladaptive motives for becoming a teacher. Journal of Education for Teaching, 38(1), 3-19. https://doi.org/10.1080/02607476.2012.643652

Fokkens-Bruinsma, M., \& Canrinus, T. E. (2014). Motivation to become a teacher and engagement to the profession: Evidence from different contexts. International Journal of Educational Research, 65, 65-74.

https://doi.org/10.1016/j.ijer.2013.09.012

Heinz, M. (2015). Why choose teaching? An international review of empirical studies exploring student teachers' career motivations and levels of commitment to teaching. Educational Research and Evaluation, 21(3), 258-297. https://doi.org/10.1080/13803611.2015.1018278 
Huber, P. J. (1967). The behavior of maximum likelihood estimates under nonstandard conditions. Proceedings of the Fifth Berkeley Symposium on Mathematical Statistics and Probability, Volume 1: Statistics, 1(1), 221-233.

Johnston, J., Mckeown, E., \& Mcewen, A. (1999). Choosing primary teaching as a career: the perspectives of males and females in training. Journal of Education for Teaching, 25(1), 55-64. https://doi.org/ 10.1080/02607479919673

Jungert, T., Alm, F., \& Thornberg, R. (2014). Motives for becoming a teacher and their relations to academic engagement and dropout among student teachers. Journal of Education for Teaching, 40(2), 173-185.

https://doi.org/10.1080/02607476.2013.869971

Keller-Schneider, M., Weiß, S., \& Kiel, E. (2018). Warum Lehrer/in werden? Idealismus, Sicherheit oder "da wusste ich nicht besseres"?: Ein Vergleich von Berufswahlmotiven zwischen deutschen und schweizerischen Lehramtsstudierenden und die Bedeutung von länderspezifischen Bedingungen. Schweizerische Zeitschrift für Bildungswissenschaften, 40(1), 217-242.

Kiel, E., Geider, F. J., \& Jünger, W. (2004). Motivation, selbstkonzepte und lehrberuf. Studienwahl und berufsperspektiven bei studierenden für das lehramt an grund-, haupt-und realschulen. Die Deutsche Schule, 96(2), 223-233.

Klassen, R. M., Al-Dhafri, S., Hannok, W., \& Betts, S. M. (2011). Investigating pre-service teacher motivation across cultures using the teachers' ten statements test. Teaching and Teacher Education, 27(3), 579-588.

https://doi.org/10.1016/j.tate.2010.10.012

Kyriacou, C., Hultgren, A., \& Stephens, P. (1999). Student teacher motivation to become a secondary school teacher in England and Norway. Teacher Development, 3(3), 373-381. https://doi.org/10.1080/13664539900200087

Lin, E., Shi, Q., Wang, J., Zhang, S. \& Hui, L. (2012). Initial motivations for teaching: Comparison between preservice teachers in the United States and China. Asia-Pacific Journal of Teacher Education, 40(3), 227-248. https://doi.org/10.1080/1359866X.2012.700047

Little, T. D., Preacher, K. J., Selig, J. P. \& Card, N. A. (2007). New developments in latent variable panel analyses of longitudinal data. International Journal of Behavioral Development, 31(4), 357-365.

https://doi.org/10.1177/0165025407077757

Little, T. D., Slegers, D. W. \& Card, N. A. (2006). A non-arbitrary method of identifying and scaling latent variables in SEM and MACS models. Structural Equation Modeling: A Multidisciplinary Journal, 13(1), 59-72.

https://doi.org/10.1207/s15328007sem1301_3

Moran, A., Kilpatrick, R., Abbot, L., Dallat, J., \& McClune, B. (2001). Training to teach: Motivating factors and implications for recruitment. Evaluation \& Research in Education, 15(1), 17-32. https://doi.org/10.1080/09500790108666980

Nóvoa, A. (1999). Os professores na virada do milênio: do excesso dos discursos à pobreza das práticas. Educação e Pesquisa, 25(1), 1-15. https://doi.org/ 10.1590/S1517-97021999000100002

Organização para a Cooperação Económica e Desenvolvimento [OCDE] (2018). Education at a Glance 2018: OECD Indicators. OECD Publishing.

Pedro, N., \& Peixoto, F. (2006). A satisfação profissional e aito-estima dos professores. Análise Psicológica, 24(2), 247-262.

http://www.scielo.mec.pt/scielo.php?script=sci_arttext\&pid=S0870-

82312006000200010

R Core Team (2013). R: A language and environment for statistical computing. R Foundation for Statistical Computing. http://www.r-project.org/ 
Rosseel, Y. (2012). lavaan: An R package for structural equation modeling. Journal of Statistical Software, 48(2), 1-36. https://doi.org/10.18637/jss.v048.i02.

Schmitz, E., \& Leidl, E. (1999). Brennt wirklich aus, wer entflammt war? Studie II: Eine LISREL-Analyse zur Burnout-Prozess bei Lehrern. Psychologie in Unterricht und Erziehung, 46, 302-310. http://www.edgar-

schmitz.de/download/brennt.pdf

Schwarzer, R., \& Jerusalem, M. (1995). Generalized Self-efficacy Scale. In J. Weinman, S. Wright \& M. Johnston (Eds.), Measures in Health Psychology: A User's Portfolio. Causal and Control Beliefs (pp. 35-37). NFER-NELSON.

Schweizer, K. (2010). Some guidelines concerning the modeling of traits and abilities in test construction. European Journal of Psychological Assessment, 26(1), 1-2. https://doi.org/10.1027/1015-5759/a000001

Sinclair, C. (2008). Initial and changing student teacher motivation and commitment to teaching. Asia-Pacific Journal of Teacher Education, 36(2), 79-104. https://doi.org/10.1080/13598660801971658

Struyven, K., Jacobs, K., \& Dochy, F. (2013). Why do they want to teach? The multiple reasons of different groups of students for undertaking teacher education. European Journal of Psychology of Education, 28(3), 1007-1022. https://doi.org/10.1007/s10212-012-0151-4

Watt, H. M. G., \& Richardson, P. W. (2008). Motivations, perceptions, and aspirations concerning teaching as a career for different types of beginning teachers. Learning and Instruction, 18(5), 408-

428. https://doi.org/10.1016/j.learninstruc.2008.06.002

Watt, H. M. G., \& Richardson P. W. (2012). An introduction to teaching motivations in different countries: comparisons using the FIT-choice scale. AsiaPacific Journal of Teacher Education, 40(3), 185-197.

https://doi.org/10.1080/1359866X.2012.700049

Watt, H. M. G., Richardson, P. W., Klusmann, U., Kunter, M., Beyer, B., Trautwein, U., \& Baumert, J. (2012). Motivations for choosing teaching as a career: An international comparison using the FIT-choice scale. Teaching and Teacher Education, 28(6), 791-805. https://doi.org/10.1016/j.tate.2012.03.003

Weiss, S., \& Kiel, E. (2013). Who chooses primary school and why?. Issues in Educational Research, 23(3), 415-433. http://www.iier.org.au/iier23/weiss.pdf 
Informação dos autores:

i Centro de Investigação e Inovação em Educação, Escola Superior de Educação, Instituto Politécnico do Porto, Portugal. https://orcid.org/0000-0002-1782-8897

ii Instituto Universitário da Maia, Maia, Portugal; Centro de Investigação e Inovação em Educação, Porto, Portugal.

https: //orcid.org/0000-0003-3927-3818

iii Centro de Investigação e Inovação em Educação, Escola Superior de Educação, Instituto Politécnico do Porto, Portugal.

https://orcid.org/0000-0002-6446-0845

iv Escola Superior de Educação, Instituto Politécnico do Porto, Porto, Portugal; Faculdade de Psicologia e de Ciências da Educação da Universidade do Porto, Porto, Portugal.

https://orcid.org/0000-0002-2884-2547

v Escola Superior de Educação, Instituto Politécnico do Porto, Portugal.

vi Ludwig-Maxmilians-University of Munich, Lehrstuhl für Schulpädagogik, Munique, Alemanha.

vii Ludwig-Maxmilians-University of Munich, Lehrstuhl für Schulpädagogik, Munique, Alemanha.

viii Pädagogische Hochschule Zürich, Zurique, Suiça. https://orcid.org/0000-0002-4442-2825

Toda a correspondência relativa a este artigo deve ser enviada para:

Sílvia Barros

Instituto Politécnico do Porto, Escola Superior de Educação, Rua Dr. Roberto Frias, 602, 4200-465 Porto.

silviabarrosaese.ipp.pt

Recebido em 1 de agosto de 2019

Aceite para publicação em 17 de novembro de 2020 
Choosing a teaching career in Portugal: Motives and self-efficacy of master students

\begin{abstract}
This study analysed students' motives for choosing teaching as a career and its associations with sociodemographic variables, teacher education program characteristics, and self-efficacy. Participants were 183 student teachers (86\% female) enrolled in different Portuguese Teacher Education Programs (master degree). Participants completed an on-line survey on their sociodemographic information and motives for their decision to become a teacher, and self-efficacy beliefs. The factorial analysis indicated four factors underlying the motives to become a teacher: (a) Characteristics of the profession, (b) Social influences, (c) Interest in the course or academic content, and (d) Promoting children's development. We found that, compared to other motives, students tend to attribute higher value to motives associated with promoting child development and the interest in the course/content. We also found statistically significant differences between the motives of students who chose a course that will allow them to teach in pre-school and/or elementary school (1st to 4th year) and those who chose a course that will allow them to teach specific subjects, as well as effects of the students' age, gender and self-efficacy. Implications for the training of teachers are discussed.
\end{abstract}

Keywords: Teaching; Intrinsic motives; Extrinsic motives; Teaching career 


\section{Elegir una carrera docente en Portugal: Motivaciones y autoeficacia de los estudiantes de máster}

\section{RESUMEN}

En este estudio analizamos las razones para que los estudiantes elijan la carrera docente y sus asociaciones con las variables sociodemográficas, las características del curso de formación docente y las creencias de autoeficacia. Participaron 183 estudiantes (86\% mujeres) de cursos de maestría en enseñanza en instituciones portuguesas, que completaron un cuestionario en línea sobre características sociodemográficas, motivaciones para su decisión de convertirse en maestros y creencias de autoeficacia. A través del análisis factorial, se derivaron cuatro factores de las motivaciones para ser maestro: (a) Beneficios de la profesión, (b) Influencias sociales, (c) Interés en el curso o contenido académico, y (d) Promoción del desarrollo de niños/adolescentes. Se encontraron valores medios más altos en las motivaciones relacionadas con la promoción del desarrollo de niños/adolescentes y el interés en el curso/contenido académico. Se encontraron diferencias estadísticamente significativas entre las motivaciones para los estudiantes que optaron por un curso para enseñar en educación preescolar y/o primer ciclo y aquellos que optaron por un curso para enseñar materias específicas, así como según edad, género y autoeficacia. Se discuten las implicaciones para la formación de profesores.

Palabras-clave: Enseñanza; Motivaciones intrínsecas; Motivaciones extrínsecas; Carrera docente 